\title{
Programa de alta hospitalaria programada para preparar el egreso del paciente $y$ familia ${ }^{1}$
}

\author{
Zeidy Vargas Bermúdez ${ }^{2}$ \\ Rebeca Fernández Coto ${ }^{3}$
}

Institución: Hospital Dr. Rafael Angel Calderón Guardia. San José, Costa Rica.

\section{COMO CITAR}

Vargas B., Fernández C.,(2011) Programa de alta hospitalaria programada para preparar el egreso del paciente y familia. Rev. Enfermería Actual

Enfermería Actual en Costa Rica [en línea].21, 1-20 [citado (fecha)]. Disponible World World Wide Web: <http://www.revenf.ucr.ac.cr/programa.pdf /ISSN 1409-4568

\section{RESUMEN}

Este artículo describe la implementación y desarrollo del Programa del Alta Hospitalaria Programada (PAH), específicamente en el área de cirugía del Hospital Rafael Angel Calderón Guardia, con la finalidad de mostrar un modelo a implementar para otras instituciones hospitalarias nacionales e internacionales, ya que proporciona herramientas a los pacientes y a sus familiares para llevar a cabo el autocuidado y su posterior reincorporación a la comunidad de la cual forman parte, partiendo de las necesidades mostradas durante su hospitalización y al momento del alta hospitalaria. Se trata de un estudio cuantitativo y descriptivo, con una población conformada por el total de egresos registrados, durante el año 2010, en el alta programada del área de Cirugías, del Hospital Rafael Ángel Calderón Guardia, seleccionando una muestra de 1224 pacientes, quienes requirieron, por parte del profesional de Enfermería responsable , valoración e intervención en la preparación del egreso. En este programa se realiza una valoración de las necesidades del paciente, tanto al momento del ingreso como la condición de egreso. La información recopilada se registra en el Instrumento de Valoración de Necesidades del paciente, el cual constituye la base para elaborar el plan de intervención para el usuario y su familia durante el internamiento y el día del egreso, orientado hacia la preparación del alta por parte del profesional en enfermería. Dicha intervención queda documentada en el Instrumento de registro de la condición de egreso de los usuarios de cirugía y en la nota de enfermería. El paciente y la familia logran, de acuerdo a la intervención del profesional de enfermería encargado del programa y con la colaboración de otros miembros del equipo de salud, adquirir conocimientos y técnicas de acuerdo al grado de dependencia o condición de salud para lograr el autocuidado al egresar del centro hospitalario. Conclusión. Los beneficios que se evidencian en la implementación del PHA giran en torno a: una preparación oportuna, según sea el grado de dependencia y de acuerdo a la condición de salud, para el autocuidado del paciente por parte de la familia al momento de egresar del centro hospitalario, una coordinación con la red externa de salud y la reubicación oportuna de los usuarios con problemas sociales, así como una agilización de los trámites administrativos.

Palabras clave: Alta-hospitalaria, Egreso, Enfermería, Familia, Paciente.

\footnotetext{
${ }^{1}$ Fecha de recepción: 13 de agosto 2011

Fecha de aceptación: 16 de setiembre 2011

2 Enfermera. Magíster en Administración de Servicios de Salud. Supervisora de Enfermería del Hospital Dr. Rafael Angel Calderón Guardia. Correo electrónico: zeidyvargas@gmail.com.

${ }^{3}$ Licda. en Enfermería. Coordinadora del Programa de Alta Hospitalaria Programada del Área de Cirugías del Hospital Dr. Rafael Ángel

Calderón Guardia. Correo electrónico: rbk.fc@hotmail.com
} 


\title{
Revista Electrónica Enfermería Actual en costa Rica
}

\section{Hospital discharge program scheduled to prepare the exit of the patient and family ${ }^{4}$}

\author{
Zeidy Vargas Bermúdez \\ Rebeca Fernández Coto 6
}

Institution: Hospital Dr. Rafael Angel Calderón Guardia. San José, Costa Rica.

\section{Cited}

Vargas B., Fernández C., (2011) Hospital discharge program scheduled to prepare the exit of the patient and family. [On line] Rev. Enfermería Actual en Costa Rica, 21, 1-20 [cited (date)]. Available World Wide Web: http://www.revenf.ucr.ac.cr/programa.pdf ISSN 1409-4568

\begin{abstract}
This article describes the implementation, development and evaluation of the Hospital Discharge Schedule (PAH), specifically in the area of surgery of the Dr. Rafael Calderón Guardia Hospital, in order to show a model to implement for other national and international hospitals, providing tools for patients and families to perform self-care and their incorporation into the community to which they belong, based on the needs shown during hospitalization and at discharge from hospital. This is a descriptive and quantitative study, whose population consisted of the total expenses for the planned discharge area of Surgery, Hospital Rafael Angel Calderon Guardia, in 2010, selecting a sample of 1224 patients who required assessment and involvement in the preparation of discharge by the nurse program manager. The program performs a needs assessment of the patient at admission and discharge status, this information is recorded in the Instrument Needs Assessment of the patient, which constitutes the basis for the intervention plan for the user and family placement and the day of discharge, preparation of oriented high by professionals in nursing. Such intervention is documented in the instrument recording the condition of graduation from users of surgery and the nursing notes. The patient and family achieve according to the intervention of the nurse in charge of the program and collaboration with other health team members acquire knowledge and skills according to the degree of dependence or health condition to achieve self-care after discharge from the hospital. Conclusion. The benefits are evident in the implementation of PHA result in a timely preparation according to the degree of dependence and according to the health condition of the patient self-care and family after discharge from the hospital, and streamline administrative procedures and the relocation users with timely social issues.
\end{abstract}

Keywords: Family, Hospital-discharge, Nursing, Patients.

\footnotetext{
${ }_{5}^{4}$ Date of receipt: August 13, 2011

Date of acceptance: September16, 2011

${ }^{5}$ Enfermera. Magister en Administración de Servicios de Salud. Supervisora de Enfermería del Hospital Dr. Rafael Ángel Calderón Guardia. Correo electrónico: zeidyvargas@gmail.com.

${ }^{6}$ Licda. en Enfermería. Coordinadora del Programa de Alta Hospitalaria Programada del Área de Cirugías del Hospital Dr. Rafaél Ángel Calderón Guardia. Correo electrónico: rbk.fc@hotmail.com
} 


\section{INTRODUCCIÓN}

Los programas de alta hospitalaria (PAH), están conformadas por una serie de estrategias desarrolladas por los y las profesionales de Enfermería, que tienen como propósito principal brindar herramientas a las personas y sus familias a cerca de los cuidados en el hogar asociados a su problema de salud.

De esta manera, a nivel internacional, se identifican programas y estudios que apoyan la orientación y seguimiento del cuidado en el hogar, por ejemplo Willins (2008), realizó un estudio del seguimiento y fortalecimiento de la red de apoyo a la familia que egresaban de las unidades de cuidado neonatal, e identificó como el diseño del programa del alta para este tipo de usuarios facilitaba la transición del hospital a la casa y tenía un impacto positivo en el costo de efectividad del mismo.

Por otra parte García (2004), refiere que si bien todo usuario tiene el derecho de recibir la información oportuna tras su proceso asistencial, se debe ser más sensible ante determinados grupos en los cuales se identifican mayores problemas de cuidados tras su alta hospitalaria.

Un estudio de Yea-Ing, (2008), realizado con un grupo de cuidadores donde la enfermera le brindó educación sobre el cuidado en el hogar al egreso hospitalario, concluyó que dicha acción aumenta la preparación de cuidadores y la satisfacción de sus necesidades. Este mismo aspecto Jack et. al (2009), mediante un estudio radomizado en donde, una enfermera del alta y un farmacéutico clínico, trabajaron con los individuos durante su hospitalización para concertar citas de seguimiento, confirmar la reconciliación de medicación y realizar educación al paciente, esto con la ayuda de un folleto de instrucciones individualizadas, enviado a su proveedor de atención primaria, y llamando a los pacientes de 2 a 4 días para reforzar el plan de cumplimiento y revisar los medicamentos, produjo una reducción en la consulta a los hospitales por parte de los pacientes y familiares posterior a su egreso.

A sí mismo, Pardo (2008) determinó que es posible desarrollar un mejor servicio de atención mediante un programa de información de alta de hospital, esto con el fin de lograr un alto nivel de satisfacción y seguridad con respecto al uso de medicamentos en el hogar.

Mediante el meta-análisis de estudios randomizados, controlados y relacionados con la efectividad de los planes de preparación del alta en ancianos, Parker (2005) concluye que dicha preparación reduce los reingresos, la hospitalización y mejoran la calidad de vida. Además, determinó que la preparación del alta es una cuestión clave para garantizar la transparencia y seguridad efectiva de las personas mayores entre los servicios de hospitalización y la atención comunitaria. Dicho investigador observó como, en la red de evidencia en salud, los informes incluyen cuatro tipos principales de intervención: la evaluación geriátrica integral, la planificación del alta, la descarga de apoyo y la educación. La evidencia presentada mostró que las intervenciones efectivas y seguras, fueron las entregadas a través de los hospitales, en coordinación con la comunidad, y esta asocia con una reducción en la tasa de reingresos al centro hospitalario, donde intervienen predominantemente los equipos multidisciplinarios, con los principios de la valoración geriátrica integral, a cargo principalmente de una enfermera especialista, usando protocolos definidos y empoderando a los pacientes utilizando métodos educativos. 


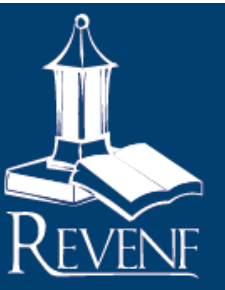

\section{Revista Electrónica Enfermeria Actual en costa Rica}

En esta misma línea, Naylor (2011), elaboraró un ensayo clínico aleatorio, dirigido a estudiar los efectos de un protocolo de planificación completa del alta, fue diseñado específicamente para los ancianos e implementado por enfermeras especialistas, con el fin de determinar los resultados en los pacientes y proveedores. El estudio incluyó las siguientes variables: estancia hospitalaria inicial, duración de tiempo entre las tasas de rehospitalización y readmisión, alta hospitalaria inicial, gastos por atención (hospitalización inicial, rehospitalizaciones), servicios de salud después de la aprobación de la gestión y servicios de enfermería especializados, evidenció que desde el alta hospitalaria inicial a 6 semanas después de la aprobación de la gestión, los pacientes en el grupo de intervención médica tenían menos reingresos y días total de readmisión hospitalaria y tasas más bajas para los servicios de atención médica, después de la aprobación de la gestión.

De esta manera, se aprecia una gran cantidad de investigaciones de alta calidad que respaldan la implementación de los programas de alta hospitalaria (PAH). Esta realidad no es ajena a Costa Rica, donde se han desarrollado algunas experiencias significativas.

Se identifica como antecedente el primer modelo nacional de preparación para el paciente y la familia para la atención al egreso hospitalario, el cual se enfocaba en el abordaje del paciente adulto mayor. Este se desarrolló como parte del programa de Visita Domiciliaria del Servicio de Geriatría del Hospital Rafael Ángel Calderón Guardia, constituidos por un equipo multidisciplinario, y actualmente referente del PAH.

Este programa (PAH) inicia su funcionamiento a partir del año 1997 en el área de medicinas, alcanzando un gran éxito, evidenciado por la disminución de reingresos y complicaciones en los pacientes. Las autoridades hospitalarias observaron que en otras áreas del hospital se presentaba problemáticas similares con respecto al egreso del paciente, por lo cual deciden, de acuerdo a informes de producción y labor presentados a la dirección de enfermería, para el 2002 extender el programa a las áreas de cirugía; para el 2004 al servicio de emergencias, y en el 2008 a la unidad de neurociencias. Lo anterior fortaleció en gran medida la experiencia en la preparación del paciente y familia para su retorno al hogar y comunidad luego del alta hospitalaria.

Parte de la riqueza del PAH se vincula a las peculiaridades que tiene el Hospital Calderón Guardia, ya que este tiene como área de atracción toda la zona atlántica, donde en los últimos años se ha carecido de anestesiólogo por lo que casi todas las cirugías son realizadas en este hospital, y son luego egresados o trasladados al hospital correspondiente.

El Programa de Alta Hospitalaria Programada (PAH), del Hospital Rafael Ángel Calderón Guardia (HCG), se creó en respuesta a las necesidades detectadas por profesionales de Enfermería, que se desempeñan como coordinadores y jefes de área de medicinas. Ellos observaban, en pacientes que egresaban de los servicios de medicina, un alto grado de dependencia para su autocuidado por su estado de salud, consecuente a patologías crónicas y complicaciones asociados a la permanencia en cama, como lo son las ulceras por presión. Estas situaciones producían angustia o temor en los familiares cuando se les comunicaba que el usuario tenía egreso y no eran incluidos en los programas de visita domiciliaria que ofrecía el hospital.

Se observaba que muchos de estos familiares no se acercaban en forma temprana y oportuna para hacer 


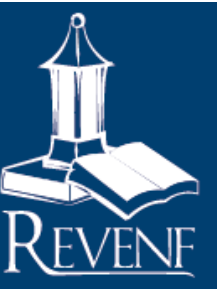

\section{Revista Electrónica Enfermeria Actual en costa Rica}

efectiva la salida del usuario, aumentando la estancia hospitalaria por problemas sociales y las complicaciones asociadas a la hospitalización prolongada. Esta problemática requería la intervención del profesional de Enfermería para preparar del paciente y su familia para el cuidado del usuario en su hogar.

El programa ha sido enriquecido al vincular la red interna del hospital, mediante el sistema de interconsultas para la preparación del egreso, con la participación de profesionales en Trabajo Social, Nutrición, programas de Enfermería, entre otros, según sean las características físicas, patológicas, socioeconómicas y de dependencia de los usuarios en su autocuidado.

El propósito de este artículo es describir el desarrollo, implementación y evaluación del PAH, específicamente en el área de cirugía del Hospital Dr. Rafael Calderón Guardia, con la finalidad de que sea un modelo para otras instituciones hospitalarias nacionales e internacionales. Además se muestra cómo enfermería interviene con los pacientes y familias, para llevar a cabo el autocuidado y su reincorporación a la comunidad de la cual forman parte, partiendo de las necesidades mostradas durante su hospitalización y al momento del alta hospitalaria.

El objetivo general del estudio fue describir la experiencia del desarrollo un Programa de alta hospitalaria programada, en el servicio de cirugía del Hospital Dr. Rafael Calderón Guardia, proporcionando medidas de autocuidado en el hogar y comunidad de acuerdo con las necesidades y recursos del paciente y familia.

\section{MATERIALES Y METODOS}

La población estuvo conformada por el total de egresos registrados en el alta programada, del área de Cirugías del Hospital Rafael Ángel Calderón Guardia para el año 2010, la cual fue de 4570 pacientes. Se determinó una muestra de 1224 individuos, seleccionada a conveniencia, dentro de aquellos que requirieron intervención directa del profesional de Enfermería responsable del programa, se tomó en cuenta el grado de dependencia que mostró el paciente para el logro de su autocuidado, asociado a su problema de salud, el apoyo familiar y de la comunidad, así como las necesidades para realizar algún procedimiento en el hogar.

Debido al gran número de pacientes que se egresan del área de cirugía, el profesional a cargo del programa, determinó criterios de inclusión de la población meta, para establecer su grado de intervención directa en el proceso, tomando en cuenta la complejidad del mismo.

La intervención directa se define, como el momento en el que el profesional de Enfermería realiza toda la preparación del paciente y familia para su egreso, tomando en cuenta las necesidades de autocuidado y trámites administrativos requeridos. Los usuarios que requirieron dicha intervención cumplieron con las siguientes características.

- Egreso por traslado a otras instituciones.

- Orientación e instrucción en la aplicación de tratamientos especiales (quimioterapia, anticoagulantes, insulina, entre otros).

- Instrucción en procedimientos como: curaciones, cambio de posición, pañales, nutrición, entre otros. 
- Baño asistido.

- Síndromes de inmovilizaciones leve o severa.

- Inmovilizaciones terapéuticas (yesos, férula)

- Evidencia de problemas familiares o sociales

- Presencia o riesgo de úlceras por presión

- Heridas abiertas o infectadas.

- Presenta ostomía

- Egresa con algún tipo de dispositivo invasivo como: sondas vesicales, sonda nasogástrica, tutores, drenos, sonda en T, VAC, entre otros.

- Necesidades nutricionales especiales.

- Instrucción especial sobre su condición de salud o procedimiento quirúrgico.

- Algún grado de dependencia para su autocuidado.

- Suministro de material para realizar procedimientos en el hogar.

El diseño se fundamentó en los lineamientos de la investigación cuantitativa, transeccional, retrospectivo, descriptivo. (Alvarado, 2008, pp.34-39,80-88)

Para la recolección de esta información, se aplicó una matriz de datos con las siguientes variables de interés: traslado a otras instituciones, interconsultas y epicrisis de enfermería, tramite de citas, instrucción al paciente y familia, trámites administrativos, procedimientos de enfermería, rondas de detección y valoración de ulceras por presión, usuarios referidos a la red extrahospitalaria y notas de enfermería.. También se utilizó la información estadística recopilada por el coordinador del programa.

La matriz de datos constó de una tabla con los registros de las distintas intervenciones realizadas por el profesional a cargo, en concordancia con las actividades generales relacionadas con el egreso, entre las cuales se agrupan las siguientes:

- Valoración de las necesidades actuales y potenciales del paciente, desde el ingreso a los servicios hasta su egreso, para realizar la programación de las intervenciones con el paciente, familia y las coordinaciones con otros profesionales de apoyo.

- Evaluar la complejidad del egreso, para determinar si asume su orientación en forma integral, y así delegar los de menor complejidad al personal de enfermería de apoyo

- Recibir del médico los expedientes de los pacientes que tienen indicado el egreso, para verificar en conjunto que todos los requisitos y documentación estén completos y tengan las indicaciones claras.

- Identificar en la familia al cuidador principal para realizar con él o ella capacitación, y entregar el programa a los familiares o cuidadores para que se presenten a recibir la instrucción, cuando el paciente sea adulto mayor, menor de edad o no se encuentra en condición para realizarse su auto cuidado.

- Brindar recomendaciones de cuidados posoperatorios.

- Tramitar la entrega del material de apoyo con los diferentes departamentos, de acuerdo con las necesidades (equipo estéril, oxígeno).

- Realizar el proceso de instrucción apoyándose en la metodología de modelaje, repetición y entrega del material didáctico correspondiente.

- Confeccionar y enviar las interconsultas a los diferentes profesionales de salud que conforman la red intra 


\section{Revista Electrónica Entermeria actual en costa Rica}

hospitalaria, como lo serían Nutrición, Trabajo Social, Ostomizados, Soporte Nutricional, Anticoagulados, Clínica de Hipertensión Arterial, Clínica de Diabéticos, Geriatría, Clínica del Dolor, entre otros.

- Comentar al médico tratante y la trabajadora social los casos difíciles de egreso, y los avances de los conocidos.

- Referir al encargado de enfermería del área de salud o red externa, aquellos pacientes que por su condición requieran de seguimiento en la comunidad, utilizando para esto la vía telefónica. Además de realizar el envió de una epicrisis y referencia detallada.

- Ejecutar los requisitos de egreso de pacientes a otras instituciones y coordinar su traslado con el Servicio de Transportes, hacer la nota y epicrisis de enfermería.

- Tramitar las citas de los pacientes que viven fuera del Área Metropolitana o que no cuentan con familiares que realicen la gestión.

- Participar en la valoración del riesgo de ulceración de los pacientes hospitalizados en el área, para identificar los ulcerados e incluirlos dentro del programa.

- Realizar procedimientos de enfermería a usuarios con egreso según sea la necesidad ( retiro de: sondas o vías, suturas, cambio de VAC, aspirar hemovack ,curaciones, aplicación de tratamientos , entre otros)

- Llenar la hoja de egreso y realizar la nota de enfermería de los pacientes que instruye.

- Realizar un control de tiempo del subproceso de egresos de los usuarios mediante el registro, en conjunto con el personal secretarial, del lapso trascurrido desde que el médico entrega el expediente con la indicación de egreso, es revisado por la enfermera, enviado a la farmacia, hasta el momento en que es trasladado a admisión para su egreso.

- Agilizar los trámites administrativos, mediante la coordinación constante con enfermeros, auxiliares de enfermería, secretarios y asistentes de pacientes.

- Entregar en forma completa, al usuario que tienen traslado al INS (vía telefónica), y solicitar la cama y coordinar con validación de derechos la priorización al trámite administrativo

- Verificar que el usuario reciba la instrucción por parte del personal de enfermería, así como de la farmacia y que lleve su tratamiento completo de acuerdo a indicación médica.

- Referir a los pacientes que egresan con ulceras por presión a la red de enfermería externa para su seguimiento.

- Elaborar el registro de usuarios atendidos en el programa.

- Elaborar material didáctico para su gestión.

La información fue procesada utilizando el programa informático EXCEL, posteriormente se realizaron tablas para su análisis.

\section{Consideraciones Éticas}

La implementación del programa del alta hospitalaria en el área de cirugía es del conocimiento y aval de las autoridades del hospital. Constituye un modelo de participación tomando en cuenta los cuatro principios de la bioética: beneficencia, no maleficencia, autonomía y justicia, ya que mediante la intervención del profesional se logra que el paciente y su familia potencialicen las capacidades para su autocuidado, de una forma voluntaria y participativa. El principio de autonomía, según los describe González (2010), consiste en la obligación de respetar los valores y opciones personales de cada individuo en aquellas decisiones que le atañen vitalmente. El programa contribuye también con los principios de justicia colectiva y oportunidad de servicios al agilizar el egreso o la 


\section{Revista Electrónica Enfermería Actual en costa Rica}

ubicación del usuario en la comunidad, impactando así en el giro cama, favoreciendo el ingreso a otro usuario a los servicios asistenciales de salud.

\section{RESULTADOS}

Las principales intervenciones directas, de acuerdo con los criterios de inclusión descritos anteriormente, que fueron realizadas por el profesional de enfermería encargado del programa se resumen en la tabla 1.

Tabla 1

Hospital Dr. R. A. Calderón Guardia: Distribución de pacientes que han participado en el Programa de Alta Hospitalaria según actividades específicas realizadas por Enfermería en el área de cirugía. 2010

\begin{tabular}{l|l}
\hline Descripción de Actividad & No. de pacientes \\
\hline Traslados realizados a otras instituciones & 177 \\
\hline Instrucciones a pacientes y familiares & 1224 \\
\hline Tramite de citas & 288 \\
\hline Procedimientos realizados al paciente con egreso & 1344 \\
\hline Interconsultas & 1388 \\
\hline Epicrisis de enfermería & 177 \\
\hline Notas de enfermería & 1286 \\
\hline $\begin{array}{l}\text { Rondas de detección y valoración de ulceras por } \\
\text { presión }\end{array}$ & 100 \\
\hline $\begin{array}{l}\text { Usuarios referidos a red de Enfermería extra } \\
\text { hospitalaria }\end{array}$ & 12 \\
\hline Revisión de trámites administrativos. & 4570 \\
\hline
\end{tabular}

La intervención el profesional de enfermería inicia con la identificación de necesidades de acuerdo a la condición física, patológica y social del usuario y familia, desde el ingreso hasta la situación presentada al momento del egreso. Utiliza el Instrumento de Valoración de Necesidades del paciente (anexo 2), el cual es la base para la elaboración del plan de atención de enfermería que requiere, esto de acuerdo al grado de dependencia para su autocuidado, características patológicas, tipo de cirugía, u otra condición que amerite la orientación para el cuidado, y coordina con el paciente y la familia el tiempo idóneo para dicha instrucción, e inicia la elaboración de las interconsultas requeridas.

Del total de la muestra, a 177 pacientes se les coordinaron traslados a otras instituciones de salud, básicamente por área de atracción o para continuar con su tratamiento o rehabilitación, de acuerdo al criterio del médico tratante, distribuidas de la siguiente forma: Hospital Tony Facio de Limón 54 pacientes, Hospital de Guápiles 36, al Instituto Nacional de Seguros (INS) 32, Centro Nacional de Rehabilitación (CENARE) 3. Se ubicaron un total de 24 pacientes en albergues por problemas sociales, asociados principalmente a falta de apoyo familiar. Cada una de las coordinaciones requiere la intervención del profesional, aspectos tales como la solicitud 
de la cama y la presentación del paciente al encargado, trámite del servicio de ambulancia, y el funcionario que lo acompaña, explicación de los requisitos a los familiares, elaboración de epicrisis y nota de enfermería.

Con respecto a "Instrucciones a pacientes y familiares", según se muestra en la tabla 2, 1224 pacientes y sus familiares requirieron instrucción sobre trámites de egreso, así como sobre cuidados generales en el hogar, asociados a su condición de salud, grado de dependencia para su autocuidado, tratamientos especiales, uso de dispositivos especiales y cuidados posquirúrgicos, lo que constituye la mayor intervención del profesional de enfermería responsable del PAH en el área de cirugías. Cada uno de los pacientes requirió, al mismo tiempo, de información sobre diferentes temas de los señalados.

Tabla 2

Hospital R. A. Calderón Guardia: Distribución del tipo de instrucción que ofrece el profesional de enfermería a pacientes y familiares, según necesidad, área de cirugía. 2010

\begin{tabular}{l|c}
\hline \multicolumn{1}{c|}{ Tipos de Instrucción } & No. pacientes \\
\hline Cuidados de sonda en T & 8 \\
\hline Cuidados de Drenos & 26 \\
\hline Cuidados de Terapia de VAC & 28 \\
\hline Cuidados de Traqueotomía & 10 \\
\hline $\begin{array}{l}\text { Aplicación de Medicamentos Vía Subcutánea. (Anticoagulante, } \\
\text { analgésico, hipoglicemiante, })\end{array}$ & 124 \\
\hline Cuidado de Drenaje urinario(Sondas) & 124 \\
\hline Cuidados de tutores & 223 \\
\hline Cuidados de Fijaciones Externas & 28 \\
\hline Cuidados de YESO & 19 \\
\hline Curación de ulceras. & 21 \\
\hline Cuidados de Hemovack & 137 \\
\hline Cuidados de Colostomía & 56 \\
\hline Cuidados de Ileostomías & 8 \\
\hline Cuidados de Yeyunostomias & 12 \\
\hline Recomendaciones a paciente Gastrectomizado. & 42 \\
\hline Síndrome de Inmovilización & 60 \\
\hline Exámenes especiales & 79 \\
\hline Tramite de oxigeno domiciliar y cuidados asociados. & 72 \\
\hline
\end{tabular}

Con relación al trámite de citas, cuando el usuario presenta dificultad al desplazarse a las diferentes consultas, por la condición de salud o en ausencia del acompañante, al igual que los egresados a otras instituciones y los traslados en ambulancia, los trámites son realizados por el personal de enfermería del servicio, previo a que egrese el paciente. Para el año 2010 se registraron un total de 288 gestiones, distribuidas de la 


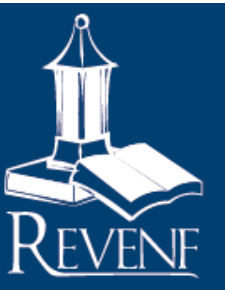

siguiente forma: de seguimiento a oncología 63 pacientes, control en rayos X 72, ortopedia 52. En el centro de equipo se tramitaron un total de 98 citas, ya que en este departamento se gestiona el material estéril requerido para que al paciente se realice algún procedimiento en su casa de habitación, como lo son la curación de heridas, ulceras, traqueotomías, entre otras. La cantidad solicitada cubre un mes, mientras realiza el trámite en la clínica o Ebais en la red de apoyo externo adscrito, cuando requiere más material.

El responsable del PAH ejecuta diariamente actividades relacionadas con procedimientos de Enfermería, registrando un total de 1344 en el año 2010. Los mismos son realizados al momento de la instrucción programada al paciente y familiares o el día del egreso cuando se revisan en la nota las indicaciones médicas. Los procedimientos más frecuentes son los siguientes: suspensión de vías periféricas o centrales , dreno, grapas, sondas, realización de curaciones, colocación o cambio de esponjas en la Terapia de Cicatrización al Vacío (Vac), aplicación de medicamentos de uso subcutáneo,(hipoglucemiantes, anticoagulantes o analgésicos).

El sistema de interconsulta es una estrategia de apoyo y abordaje multidisciplinario e interdisciplinario, que utiliza el encargado del PAH al identificar las necesidades del usuario. Esta actividad consiste en realizar, en forma escrita, una descripción de la situación presentada por el paciente y el motivo de la interconsulta, para que el referente lo conozca y realice su intervención. Este procedimiento se inicia desde el momento del ingreso del paciente y puede requerir varias interconsultas de acuerdo a la patología, complicaciones o necesidades manifestadas durante la hospitalización. De acuerdo con el profesional que interviene en la atención del paciente, dicho abordaje se convierte en seguimiento, complemento, refuerzo, de apoyo o independiente a la realizada por el encargado del programa. De acuerdo a los datos registrados en el programa, en el año 2010 se realizaron un total de 1388 interconsultas, distribuidas de la siguiente forma:

Al Servicio de Ambulancia un total de 467, para el traslado de los usuarios, principalmente a otro centro hospitalario o por condición de salud y dependencia para el autocuidado.

Al Servicio de Nutrición y Soporte Nutricional un total de 157, para instrucción sobre preparación de alimentos o tipos de dietas asociadas a patología o uso de dispositivos como sondas.

Tres al Servicio de Rehabilitación, cuando se indica traslado del paciente al CENARE o continuar fisioterapia y en el paciente que requiere movilización temprana en su unidad, posterior a la cirugía ortopédica, ya que se le brinda la instrucción al paciente y familia sobre los ejercicios a realizar durante su hospitalización y a su egreso, con el fin de fomentar la seguridad, estimular la movilización temprana, reducir el encamamiento y mejorar la calidad de vida.

Un total de 219 interconsultas se realizaron a trabajo social, al identificar algún tipo de problema familiar o social, para que se realice el estudio en forma temprana y, de requerirlo, se tramite su ubicación en albergues, ubicando para el año 2010 un total de 24 pacientes.

Se registraron un total de 149 interconsultas al centro de equipos relacionadas con la distribución de material para los procedimientos de los usuarios en el hogar. 


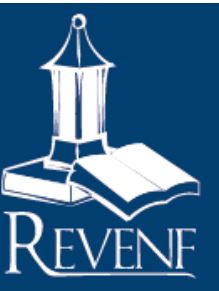

Al Departamento de Endocrino se refirieron un total de 124 usuarios, ya fuera por desarrollar un debut diabético, por el tipo de cirugía o por que, de acuerdo a la valoración del profesional de Enfermería, requerían instrucción o reforzamiento del mismo, asociado a los cuidados generales y de medicamentos para el paciente diabético.

A la Clínica de Ostomizados se realizaron un total de 44 interconsultas, para pacientes que, debido a su patología, les fue realizada la ostomía y por lo tanto requirieron ser ingresados al programa del hospital, el cual esta integrado por un equipo multidisciplinario, dedicado a llevar un control y suplir mensualmente el material necesario.

A la Clínica de Anticoagulación del hospital se refirieron un total de 11 pacientes, todos ellos egresados con tratamiento, ya sea por un periodo corto o para el resto de su vida, y por lo tanto requieren seguimiento y control, de acuerdo a los protocolos de atención establecidos.

En el segundo semestre se implementó en la institución la Consulta de Hipertensión, a la cual fueron referidos un total de 30 pacientes para su instrucción y seguimiento.

En lo referente al seguimiento del plan del alta del paciente, ya sea en las clínicas periféricas o en la red extrahospitalaria, se registraron un total de 12 interconsultas. En ellas donde se realizó la identificación vía telefónica del responsable, se le presenta el paciente y envía el plan mediante la modalidad de epicrisis, con el objetivo que se le dé la atención respectiva en su comunidad.

Al programa de Visita Domiciliar del Departamento de Geriatría, se enviaron un total de 10 interconsultas de pacientes geriátricos, con el propósito de integrarlos a dicho programa y así se les brinde el seguimiento respectivo.

Los pacientes con patologías oncológicas que requieren un abordaje integral y del dolor se refirieron, mediante interconsulta, un total de 13 al Centro Nacional del Control del Dolor y Cuidados Paliativos.

Un aspecto tomado en cuenta al realizar la valoración integral de las necesidades del usuario es la presencia de ulceras por presión, ya sea que el paciente las presente al ingreso o las desarrolle durante su hospitalización. Debido a esto, el responsable de la preparación del alta, utiliza como estrategia para la detección, valoración y seguimiento de dicho suceso adverso, la participación en las rondas de revisión de los usuarios de riesgo, con el objetivo de incluir la orientación al familiar o cuidador del paciente. En el año 2010 se realizo un total de 100 valoraciones detectando un total de 45 pacientes con presencia de ulceras por presión a su egreso distribuidas de las siguiente manera: 38 pacientes con ulceras grado I, 31 grado II y un paciente con ulcera grado III y uno con una Grado IV.

La utilización del instrumento Registro de la condición de egreso de los usuarios del servicio de cirugías, (anexo1) y la elaboración de la epicrisis de Enfermería en los casos que se refieren a otras instituciones de salud de la red de apoyo comunitario o albergue de referencia, constituyen el informe del alta, ya que el profesional de enfermería responsable registra información de la intervención realizada, así como las acciones que 


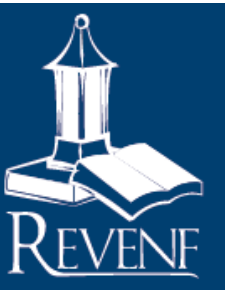

\section{Revista Electrónica Enfermería Actual en costa Rica}

deben continuarse para la atención de los usuarios. Dichos instrumentos son entregados a los pacientes y queda una copia en cada expediente de salud. La intervención realizada en la preparación del egreso se evidencia en el expediente, adjunta copia de los instrumentos descritos y con la elaboración de la nota de Enfermería al egreso a cada usuario, para el año 2010 fueron realizadas un total de 1286 por el coordinador del programa.

\section{DISCUSIÓN}

Actualmente los países industrializados y en vías de desarrollo enfrentan un incremento en los costos relacionados con los servicios de salud, principalmente los hospitalarios. Esto asociado a la complicación de la atención, al aumento de la esperanza de vida, así como a la complejidad de las enfermedades, y al avance de la ciencia en el campo de la salud. Ante este panorama los sistemas de salud crean alternativas de gestión que puedan permitir una estancia hospitalaria más corta, y una participación más activa del usuario y su familia como parte del sistema.

Costa Rica no escapa a esta realidad, ya que el comportamiento financiero del sistema de salud, la capacidad instalada de los hospitales y la demanda de servicios está en ascenso, por tal motivo se requiere desarrollar programas que estimulen y contribuyan a garantizar un giro cama expedito en la institución, garantizando de esta manera una mayor oportunidad de acceso a la población.

Algunos autores afirman que ante este panorama el alta hospitalaria precoz fue estimulada como objetivo, entre otros, para aumentar la productividad y disminuir costos, aspectos importantes desde el punto de vista administrativo.

En la década de los 80 el alta hospitalaria era frecuentemente percibida como el final triunfante de una serie de sofisticados cuidados médicos y de Enfermería, era considerado como sinónimo de suceso y de misión cumplida, no obstante, existían un gran número de pacientes que retornaban al hospital con recidivas de problemas que podrían ser prevenidos o controlados. Enfoques recientes consideran el alta hospitalaria como proceso de transferencia de responsabilidad del Cuidado a la familia y a otros profesionales de salud. (Cardozo, 2000)

Autores como Shepperd (2010) refieren como la planificación del alta, es una característica de rutina en los sistemas de salud de muchos países, cuyo objetivo principal en la planificación del alta es reducir la duración de la estancia hospitalaria y los reingresos al hospital no planificados y mejorar la coordinación de los servicios después del alta del hospital.

La preparación para el regreso del paciente al grupo familiar, a través del Programa de Alta Hospitalaria Programada, se ha constituido en un modelo que responde a esas necesidades, estableciendo dentro de sus objetivos, un punto de vista económico, reducir la estancia hospitalaria injustificada, asociado esto a problemáticas sociales y los reingresos por desconocimiento de cuidados de salud del usuario y familia, así como vincular la trasferencia del cuidado del usuario al cuidador o familiar, tomado en cuenta el apoyo de la red comunitaria. 


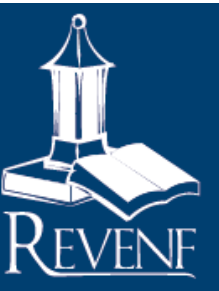

\section{Revista Electrónica Enfermeria Actual en costa Rica}

Actualmente la conceptualización de la salud involucra como actor principal al paciente y familia como responsable de su bienestar, donde el personal de salud debe centrar esfuerzos en el fortalecimiento de las potencialidades personales y colectivas.

El modelo de preparación del alta toma en cuenta que las personas deben estar informadas y educadas en salud, haciendo énfasis en el fortalecimiento de actitudes positivas que les ayuden a vivir de forma saludable. Esto es fundamental para lograr cumplir los objetivos propuestos en la creación del mismo, ya que sus estrategias de acción lo constituye el factor educativo con una presencia activa del paciente, familiar y /o cuidador.

El cuidador del paciente, ya sea un familiar directo o la persona contratada por la familia, se constituye, en este programa, en el eje fundamental del profesional de enfermería para brindarle el entrenamiento específico de acuerdo a las necesidades, con el fin de garantizar la continuidad del cuidado establecido para dicho individuo de acuerdo a sus necesidades y grado de dependencia.

El programa desarrolla aspectos como los anotados por algunos autores, tal es caso de Cardozo (2000), quien refiere a la importancia de repensar en estrategias de asistencia e implantar nuevas metodologías, buscando la atención integral del paciente, asegurando los servicios necesarios que garanticen la continuidad del cuidado, principalmente para el paciente con enfermedad o condición crónica que demanda cuidados después del alta hospitalaria, de una manera eficiente con el apoyo del equipo de salud y una buena relación de costo-beneficio.

De acuerdo con la investigacion realizada sobre el tema, se identificaron algunos modelos con caracteristicas similares, donde implementan estrategias para el seguimiento del cuidado del paciente, en coordinación con la comunidad, tomando en cuenta las necesidades de los individuos y sus familias, a la luz de las teorizantes, y el proceso de atención de enfermería, con la utilización de la tecnología en línea para agilizar la comunicación entre niveles e interinstitucional. (Hospital San Juan de Dios del Aljarafe, 2006)

Esta necesidad de articular la asistencia hospitalaria con la red comunitaria es vital para cumplir con los objetivos del programa en los pacientes y familiares que requieren completar su rehabilitacion o necesitan recursos estrahospitalarios para su autocuidado.

El paciente que egresa del área de cirugía presentan, en un gran porcentaje, necesidades asociadas a patologías agudas de resolución quirúrgica, enfermedades crónicas con patología terminal, dispositivos relacionados con las diferentes técnicas quirúrgicas, lesiones traumáticas o adultos mayores con un alto grado de dependencia para su autocuidado, lo que amerita que se realice una coordinación con las redes de apoyo, para garantizar la continuidad de los cuidados requeridos.

En ese escenario, el enfermero es identificado como el profesional apropiado para asumir la gerencia del cuidado, instrucción y orientación del paciente y la familia debido a su conocimiento clínico, a la habilidad para ofrecer cuidado holístico y abogacía, así como para realizar los trámites administrativos, esto por estar informado de los servicios que otros profesionales y personal asociado brinda. (Cardozo, 2000)

Al considerarse que un paciente puede ser egresado de un centro hospitalario no significa, desde el punto 


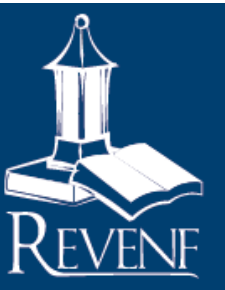

\section{Revista Electrónica Enfermeria Actual en costa Rica}

de vista de Enfermería, que dicho individuo no requiere atención para su autocuidado, sino que debe orientar el plan de atención a la etapa final del proceso hospitalario, que conlleva la preparación de ese individuo y su familia para asumir el autocuidado sin la presencia 24 horas del personal de Enfermería. Esto tiene como propósito final evitar los reingresos a nivel hospitalario y de esta manera disminuir los costos, ya de por sí elevados, de la atención en salud, especialmente lo relacionado con la cirugía. Es en esta etapa donde se culmina la preparación, con el regreso al hogar, o, en su defecto, con las coordinaciones requeridas con otras instituciones para la reubicación, ya sea para la continuidad de terapias o por problemas sociales.

Al establecer la preparación del paciente y familia para el alta, se vislumbran las necesidades, a la luz del restablecimiento del autocuidado por parte del individuo, razón por la cual el programa del alta hospitalaria se sustenta en la teorizante Dorotea Orem, cuyo modelo está constituido por acciones que la enfermera establece de acuerdo a la identificación de las necesidades, donde pretende lograr que el individuo realice su autocuidado al egresar de la institución, consecuente con la teoría que describe como la persona debe controlar los factores internos y externos que pueden comprometer su vida y su desarrollo posterior.

El autocuidado es una conducta que realiza, o debería realizar, la persona por sí misma. Según Orem (1983) no es innato, sino aprendido a lo largo de la vida, a través de las relaciones interpersonales y la comunicación en la familia, la escuela y amigos. La teoría es un análisis detallado de los cuidados enfermeros, lo cual permite definir el espacio ocupado por el profesional de Enfermería en relación con la persona, donde todos los individuos sanos adultos tienen la capacidad de autocuidado, en mayor o menor grado, en función de determinados factores (edad, sexo, estado de salud, situación sociocultural, predisposición). Independientemente de cuál sea la problemática presentada, el tipo de enfermedad, falta de recursos o los factores ambientales, la demanda de autocuidado terapéutico de la persona es superior a su agencia de autocuidado, entonces la enfermera actúa ayudando a compensar el desequilibrio a través de los sistemas de enfermería. (Rivero, 2007).

Dorothea Orem concibe al ser humano como un organismo biológico, racional y pensante; los seres humanos tienen la capacidad de reflexionar sobre sí mismos y su entorno, capacidad para simbolizar lo que experimentan y usar creaciones simbólicas (ideas, palabras) para pensar, comunicarse y guiar los esfuerzos para hacer cosas que son beneficiosas para sí mismos y para otros. La autora define los cuidados de Enfermería como el ayudar al individuo a llevar a cabo y mantener acciones de autocuidado para conservar la salud y la vida, recuperarse de la enfermedad y afrontar las consecuencias de ésta. El entorno es entendido, en este modelo, como todos aquellos factores físicos, químicos, biológicos y sociales, ya sean éstos familiares o comunitarios, que pueden influir e interactuar en la persona. Finalmente, el concepto de salud es definido como un estado que, para la persona, significa cosas diferentes en sus distintos componentes y cambia a medida que cambian las características humanas y biológicas de la persona (Navarro, 2010; Fawcett, 2001)

Para los profesionales de Enfermería es importante dominar una premisa fundamental de la teoría, la que plantea como las personas adultas tienen el derecho y la responsabilidad de cuidarse a sí mismas, así como a aquellas personas dependientes bajo su cargo. En caso de que no puedan hacerlo, deben buscar ayuda en los familiares o profesionales de la salud. Orem también tiene en cuenta la posibilidad de que la persona no quiera, por varios motivos, asumir la responsabilidad de cuidarse, como puede ser el caso de trastornos psíquicos u otras prioridades (Martínez, 2007) 


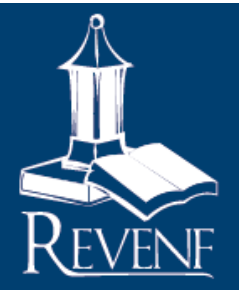

\section{Revista Electrónica Enfermeria Actual en costa Rica}

Con relación al fundamento teórico anterior y, de acuerdo a la estructura del programa de preparación para el alta, la enfermera o enfermero, desde la valoracion inicial del individuo y familia al ingreso al hospital, promueve en el paciente y su familia la adquisicion de conocimientos y estrategias que aseguren, la participación informada y voluntaria en su autocuidado, anticipando una efectiva preparacion para el egreso hospitalario; ya que, según como lo describen algunos autores refiriendose a esta teoría, el individuo debe ser capaz de participar de forma activa en la planificación e implementación de su propia atención de salud, teniendo en cuenta los factores básicos condicionantes del autocuidado, la edad, el estado de salud y los conocimientos que poseen de ésta, la pertenencia al grupo social y los hábitos del medio cultural, la falta de habilidades y hábitos, el concepto de sí mismo y la madurez de la persona. (Martinez, 2007)

Las estrategias desarrolladas en la preparación del usuario y familia para el egreso hospitalario, toman en cuenta los requisitos que las personas deben cumplir cuando se ocupan de su autocuidado. Existen tres tipos de requisitos: universales relacionados con la satisfacción de las necesidades básicas de todo ser humano, de desarrollo referentes a necesidades según el período del ciclo vital, de desviación de la salud cuando las necesidades se basan en la alteración de la salud. Ya que como lo anota Rivero (2007) el modelo de Orem promueve la satisfacción de las demandas de autocuidado del individuo, fomentando su autonomía, ya que la meta que la enfermera se plantea utilizando el modelo está dada por la asistencia a las personas para que alcancen al máximo su nivel de autocuidado.

La intervención del profesional responsable del programa inicia desde el ingreso del paciente al servicio de hospitalización, al momento de realizar la valoración inicial de la condición del individuo con las necesidades reales y potenciales a considerar. Posteriormente, tomando en cuenta el motivo de su hospitalización, se establece un plan de acción que involucra al individuo, familia y comunidad, así como los recursos complementarios, como es la participación de otros profesionales en el campo de la salud (red interna), los cuales garantizan la preparación del egreso del paciente. Incluye los aspectos físicos, patológicos, recursos económicos, factores de riesgo social, y así determinar el apoyo familiar o comunitario que eventualmente el paciente pudiese necesitar de acuerdo al motivo de ingreso.

En este aspecto todas las necesidades anticipadas son consideradas en el plan del alta, con el fin de potencializar al máximo la calidad de vida del paciente. Ya que, como refieren algunos autores, si alguno de los datos o informaciones son perdidas, el plano de alta total se torna inestable o mínimo, lo que resulta en confusión y pueden ser necesarios cambios de último minuto. El tiempo y gasto realizado en una evaluación cuidadosa y profunda puede ser tiempo valioso. (Cardozo, 2000)

La preparación del paciente y la familia para el alta es un proceso de cuidado y educación que inicia durante la hospitalizacion, y requiere una participación activa del profesional, por lo tanto es necesario desarrollar este aspecto en las instituciones de salud, para de esta manera mejorar la relación enfermera/paciente/familia, y así reducir las complicaciones asociadas a falta de instrucción sobre el cuidado. Requiere de un cambio de actitud del profesional y en los planes de atención de Enfermería, para aprovechar el momento adecuado en el paciente para que entienda, aprenda y participe en su autocuidado (Alcala, 2007)

Este plan debe garantizar la coordinación entre niveles asistenciales y la continuidad de cuidados. Debe 


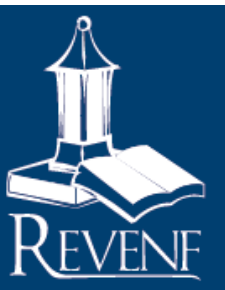

\section{Revista Electrónica Enfermería Actual en costa Rica}

informar al paciente, y a las áreas de codificación, de los cuidados realizados durante la estancia hospitalaria, así como proporcionar las recomendaciones de cuidados que requieren el paciente y la familia para dar respuesta sus necesidades. (Merino-Ruiz, 2008)

A lo largo de la hospitalización, el profesional incluye en su programación para la intervención en la preparación del individuo y familia, diferentes acciones a realizar, este tipo de intervención es conocido en otros sistemas de salud como informe del alta ( Merino Ruiz 2008 ), y constituye otro eje medular del programa, pues es la manera de garantizar la disminucion de las complicaciones prevenibles de los individuos, así como su independencia de acuerdo a la evolucion y calidad de los cuidados.

En esta temática, un estudio de Cruzado (2008), demuestra la necesidad de establecer ciertos mecanismos para garantizar la continuidad de los cuidados del paciente en el hogar u otras instituciones, de acuerdo a la experiencia de la hospitaliazacion. Esta práctica debe inmplementarse como parte de las actividades innerentes al profesinal, garantizando así una mayor oportunidad para los usuarios al egresan de las instituciones de salud.

El informe de Enfermería del alta hospitalaria es considerado como un documento de vital importancia para garantizar la continuidad de los cuidados, ya sea a través de otros profesionales sanitarios como del propio paciente y/o cuidador principal. (Ubé, 2006)

Otras propuestas basadas en esta experiencia refieren que este tipo de informes deben mejorarse, esto con el objeto de establecer mecanismos de unificacion de criterios y una proyección de Enfermería, en este aspecto, más independiente, y no solo limitarse al punto de vista de cuidados médicos, sino más bien a la planeación de las actividades desde el punto de vista de Enfermería, tomado en cuenta las necesidades de los usuarios. (Biblioteca Las Casas, 2005)

En la preparación del alta a través del desarrollo del programa, el profesional de Enfermería utiliza, como herramienta para garantizar la continuidad del cuidado del usuario, estategias de coordinacion vía telefónica y el envío de la epicrisis de Enfermería, las cuales contienen el resumen de la condición del paciente durante la hospitalalización y la descripción del plan de cuidados a continuar posterior al egreso a las instituciones gubernamentales como clínicas, Ebais e instituciones no gubernamentales (asociaciones, hogares de ancianos y alberguez), lo cual contituye la activación de la red de apoyo extrahospitalaria, de acuerdo al domicilio o destino del paciente posterior a la hospitalización. Esta conformación de la red de apoyo se ha desarrollado a través de los años, lo que constituye una de las fortalezas del programa y a contribuido al éxito del mismo. En los casos en donde se necesita apoyo para re-ubicar al usuario, el profesional de trabajo social ocupa un papel fundamental en dicha valoración y ubicación, como parte del equipo de salud.

Otro aspecto relevante del programa es el informe de Enfermería del alta, al ser este un documento de suma importancia en la actividad de la enfermera o enfermero, pues resume y estructura la información sobre las circunstancias en que se ha desarrollado el cuidado. Autores como (Alfambra, 1996; Camacho, 1998; Torres, 2000 , citado por Ruiz, 2005) lo describen como "el vínculo para evaluar los cuidados prestados por el personal de enfermería y permite la continuidad de los mismos." (p.2) Manifiesta además que este informe debe integrar cuidados dirigidos al paciente, familia (o bien cuidador informal o su red de apoyo), para lograr unos cuidados 


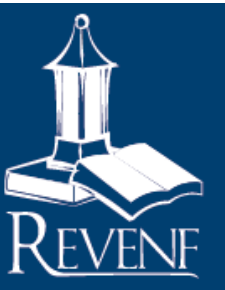

\section{Revista Electrónica Enfermería Actual en costa Rica}

domiciliarios óptimos.

Dicho autor manifiesta además la existencia de otros estudios que abogan por informes de evolución de enfermería realizados en el Centro de Salud, los cuales pueden aportar los conocimientos que Enfermería tenga de la comunidad, de la situación familiar y del propio individuo, cuando éste ingrese en un centro hospitalario; y refiere que, de acuerdo al estudio realizado, "un 90\% de los profesionales de Atención Primaria creen que el informe de Enfermería al alta les permite una buena comunicación con atención especializada" (Ruiz, 2005, p.2).

\section{Asimismo refiere que}

“el informe de Enfermería al alta no debe ser una copia de la alta médica, sino que debe complementarse y ofrecer aspectos diferentes de la hospitalización del paciente, poniéndose así de manifiesto la autonomía de dos profesiones que trabajan desde la interdisciplinariedad. Tampoco debe de convertirse en un papel más a rellenar y entrar a formar parte de una historia, ya muy voluminosa".(Ruiz, 2005, p.2)

A la luz de estas apreciaciones, el programa de preparación para el alta emite el informe de hospitalizacion durante las coordinaciones con las instituciones de referencia para la continuidad de los cuidados, ya sea por área de atracción, condición del usuario o problema social. La comunicación se establece vía telefónica y el envío del resumen de la estancia hospitalaria y la continuidad del plan de cuidados se incluye en la epricrisis de enfermería realizada por el coordinador.

En el hospital, el impacto de esta práctica se ha demostrado con la implementacion y desarrollo del programa en cuatro áreas de hospitalizacion: emergencias, medicina,cirugía y neurociencias, cada una de estas áreas atiende a individuos con necesidades especificas. Esto concuerda con los resultados documentados en los programas de preparación para el alta en países donde en los hospitales egresan pacientes con características similares a las que tienen los pacientes en nuestro medio (Abad-Corpa, 2010, Lin 2009,Yea-Ing , 2008)

En este aspecto el programa establecido para el área de cirugía abarca pacientes de diferentes especialidades quirúrgicas, como lo son oncología, urología, cirugía (tórax, recosntructiva, maxilofacial, ortopedía, otorrinolaringología, vascular periférico y cirugía general), cada uno con características muy particulares que van desde enfermedades agudas y crónicas, hasta traumatismos y secuelas muy importantes asociadas a los mismos.

Es importante señalar en este aspecto que de acuerdo a los datos estadísticos del programa, relacionados con la dinámica de los servicios, tipo de cirugías y procedimientos, muchos de los pacientes de cirugías egresan con dispositivos invasivos como: drenos, sondas, asociados al tipo de patología o la técnica quirúrgica implementada, heridas en vías de cicatrización, lo que conlleva una preparación más exaustiva en el manejo y cuidadados, con el fin de disminuir complicaciones asociadas a traumatismos, lesiones e infeciones entre las mas comunes, que le probocarían desde una incomodidad, hasta el reingreso a la institución.

El profesional de enfermería responsable del programa, además de las coordinaciones y la preparación del usuario, familia o cuidador para que participe en la asitestencia del usuario, de acuerdo a su categorización, debe 


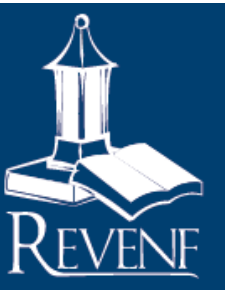

cumplir con actividades relacionadas con los requisitos administrativos del egreso, las cuales contemplan una serie de acciones y agilización de trámites en los departamentos correspondientes, tal es el caso de validacion de derechos, admisión, farmacia, entre otros. además debe controlar y ejecutar los registros de la información en los diferentes instrumentos, para la verificación de la preparación del paciente, intervención del equipo de salud y condición de egreso.

\section{CONCLUSIONES}

El Programa de Alta Programada Hospitalaria (APH) para la preparación del paciente y familia al hogar, los orienta con respecto a su autocuidado, con la finalidad de disminuir complicaciones que conduzcan hacia el reingreso al hospital.

La estructura y desarrollo del APH está sustentado en el modelo conceptual de D. Orem, en donde el profesional de enfermería desempeña el papel de apoyo sustentador. Sus acciones están basadas en los metaparadigmas: persona, salud, entorno, cuidado y rol del profesional.

Los beneficios que se evidencian en la implementación del APH redundan en una preparación oportuna del paciente para su egreso según su condición de salud, así como reubicación temprana de pacientes con problemas sociales, utilización de redes de apoyo y respuesta más efectiva de servicios institucionales complementarios para el traslado hacia el destino de egreso.

El programa disminuye los reingresos y las complicaciones asociadas a estos, lo cual significa un ahorro de recursos muy importante para la institución.

\section{REFERENCIAS}

Abad-Corpa, E. (2010) Effectiveness of planning hospital discharge and follow-up in primary care for patients with chronic obstructive pulmonary disease: research protocol. (J.compilation, Ed.)

Journal of Advance Nursing, 66(6), 1365-1370.

Alcala. P. (2007) Nurses performance hospital discharge: patient Point of view. Activa 20, 245-350.

Alvarado, E. (2008). Metodología de la Investigación (Tercera Edicion. ed.). Washington, D.C.: Organización Panamericana de la Salud.

Biblioteca Lascasas. Fundación Index (2005). Guías de Enfermería Recuperado el 10 de julio de 2011, de htpp://www.index_f.com/lascasas/lascasas.php.

Cardozo G. (2000) Gerenciamiento de caso de enfermería en el procesode alta hospitalaria del paciente con lesión medular. (S. M. Cardiológia, Ed.) Revista Mexicana de Enfermería Cardiológica, 8, 1-4, 7-13

Cruzado A. (2008) Cardiología en Enfermería, Tercer Cuatrimestre 2008 (45), 23. 


\section{Revista Electrónica Enfermería Actual en costa Rica}

Cruzado, G. (2004) Continuidad d elos Cuidados enfermeros tras alta hospitalaria. (J. d. Andalucia, Ed.) Servicio Andaluz de Salud.Consejeria de salud, 1-13.

Fawcett, J. (2001). The Nurse Theorists: 21st-Century Updates-Dorothea E. Orem. (I. Sage Publications, Ed.) Nursing Science Quarterly, 14 (1), 34-38.

González, M.(2010) Principales consideraciones bioéticas en la atención en salud sexual y reproductiva en adolescentes. (SciELO, Ed.) Revista chilena de obstetricia y ginecología, 75 (4), 272 - 277.

Hospital San Juan de Dios del Aljarafe. (2006) Informe de Continuidad de Cuidados de Enfermería al Alta ICCAE. (Saludinnova.com, Ed.) 1-26.

Jack, W., Chetty, K, Anthony, D., Greenwald L., Sanchez, G., Johnson, E., y otros. (2009). Areengineered Hospital discharge Program to decrease rehospitalizationt:a Randomized trial. (A. C. Physicians, Ed.) Annals of international Medicine ,(3)150, 178-187.

Lin P C., Wang C H., Chen Ch.,Liao L., Kao S., Wu H.,(2009). To evaluate the effectiveness of a discharge-planning programme for hip fracture patients. (J. compilation, Ed.) Journal of Clinical Nursing, 18, 1632-1639. Recuperado de http://onlinelibrary.wiley.com/doi/10.1111/j.1365-2702.2008.02695.x/full

López, T. (s.f.) Protocolo del servicio de continuidad de cuidados de Enfermería entre ámbitos asistenciales. (D. G. Salud, Ed.) Servicio Canario De Salud, 1-37.

Martínez, N. (2007) Valoración ética del modelo de Dorotea Orem,Vol.6,n3. ( Scielo Rev haban cienc méd [online].) Recuperado el 16 de julio de 2011, de <http://scielo.sld.cu/scielo.php?script=sci_arttext\&pid=S1729519X2007000300012\&lng=es\&nrm=iso $>$. ISSN 1729-519X.

Merino M, (Comunicación personal, 5 de Mayo de 2011)

Merino-Ruiz (2008) Utilidad y dificultades para la realización del informe de enfermería al alta hospitalaria según las enfermeras del Hospital de Fuenlabrada. (H. d. Fuenlabrada, Ed.) Enfermeria Clinica, 18(2), 77-83.

Naylor, M. (2011)Comprehensive Discharge Planning and Home Follow-up of Hospitalized Elders. (J. A. Journals, Ed.) Jama Archives Journals, 305 (24), 2493-2592.

Navarro P. (2010) Modelo de Dorothea Orem aplicado a un grupo comunitario a través del proceso de enfermería.. Enfermería Global (19), 1.14.

Orem, D. (1983)._Normas Prácticas en Enfermería. Madrid: Ediciones Pirámide.

Pardo L,(2008) Consultenos: programa de información al alta hospitalaria. Desarrollo y resultados del primer añode funcionamiento en 5 hospitales. Servicio de Farmacia. Hospital Universitari San Joan d'Alacant, 32(6), 323-330.

Parker, S.(2005). Do current discharge arrangements from inpatient hospital care for the elderly reduce readmission rates, the length of inpatient stay or mortality, or improve health status. (W. R. Europe., Ed.) Health Evidence Network. 


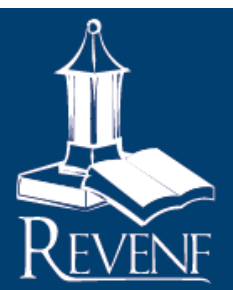

Ruiz, M.C. (2005). Utilización del diagnóstico enfermero en los informes de enfermería al alta hospitalaria. Biblioteca Lascasas, 1 Disponible en http://www.index-f.com/lascasas/documentos/lc0012.php

Shepperd S, ( 2010) Planificación del alta del Hospital al domicilio. Recuperado el 15 de julio de 2011, de Colaboración Cochrane: http://www2.cochrane.org/reviews/es/ab000313.html

Ubé, P. (2006). Evaluación de la relevancia del informe de enfermería al alta. Nure Investigación, $n^{\circ} 24,24$.

Willis V, ( S.F.) Parenting Preemies, A Unique Program for Family Support and Education After NICU Discharge. Advances in Neonatal Care • Vol. 8, No. 4 Recuperado de www.advancesinneonatalcare.org

Yea-Ing Sh, (2008). A family caregiver-oriented discharge planning program for older stroke patients and their family caregivers. (J. compilation, Ed.) Journal of Clinical Nursing , 17, 2497-2508. 